\title{
POLYNOMIAL LPV SYNTHESIS APPLIED TO TURBOFAN ENGINES
}

\author{
Wilfried Gilbert* Didier Henrion *,** \\ Jacques Bernussou* David Boyer*** \\ * LAAS-CNRS, Toulouse, France

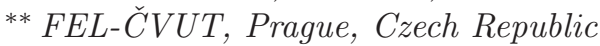 \\ *** Snecma, Villaroche, France
}

\begin{abstract}
Results on polynomial fixed order controller design are extended to SISO gain-scheduling with guaranteed stability and $H_{\infty}$ performance over the whole scheduling parameter range. Salient features of the approach are (a) the use of polynomials as modeling objects; (b) the use of flexible LMI conditions allowing polynomial dependence of the open-loop system and controller transfer functions in the scheduling parameters; and (c) the decoupling in the LMI conditions between the Lyapunov variables and the controller variables, allowing both parameterdependent Lyapunov functions and fixed-order controller design. The synthesis procedure is integrated into the ATOL framework developed by the manufacturer of aircraft and space engines Snecma to systematically design reduced complexity gain-scheduled control laws for aircraft turbofan engines.
\end{abstract}

Keywords: Turbofan engine control, linear parameter varying (LPV) systems, fixed-order controller design, linear matrix inequalities (LMI), polynomials

\section{INTRODUCTION}

Gain-scheduled control laws are widely applied to industrial plants whose dynamical properties strongly vary with the operating point or the environment. There exist various techniques for gain-scheduling, from ad hoc tuning procedures to more sophisticated techniques ensuring stability and performances over the whole operating range, see (Leith and Leithead, 2000; Rugh and Shamma, 2000) for comprehensive surveys. In the latter category we can find linear parametervarying (LPV) controller design via convex optimization over linear matrix inequalities (LMI), a methodology which has found various applications especially in the aerospace industry. In particular, as shown recently in (Balas, 2002) or (Bruzelius, 2004) these techniques can succesfully be applied to control aircraft turbofan engines, which are characterized by a strong sensitivity of the system dynamics with respect to the flight envelope (power lever angle, aircraft speed, altitude) or the environment (inlet pressure, temperature).

The main drawbacks of currently available design procedures for LPV/LMI gain-scheduling are as follows:

- a linearizing change of variables is used to ensure convexity of the LMI design conditions, and the controller must be reconstructed using tedious linear algebra, see (Scherer and Weiland, 2000) for a good overview;

- the order of the controller must be equal to the order of the plant plus the order of the weighting functions, which is contradictory with the sometimes stringent reduced complexity specifications ubiquitous in embedded aerospace control systems; 
- an appropriate state-space canonical form must be obtained over the whole operating range.

All these issues are explicitely and fairly enumerated in the recent paper (Wassink et al., 2005) where an LPV/LMI gain-scheduled control law is designed for a mechatronics system.

In this paper we propose an original gain-scheduling design procedure which is aimed at removing the above drawbacks:

- the controller variables appear explicitly as decision variables in the design LMI;

- the order of the controller as well as its structure are fixed from the outset, independently of the open-loop system order and weighting functions;

- the design conditions are directly formulated in the polynomial setting.

Our main objective is to provide a relatively simple design methodology integrated into the ATOL control design framework (Vary and Reberga, 2005) developed by Snecma ${ }^{1}$.

Earlier works resulted in a collection of linearized models at various operating points (Henrion et al., 2004) as well as LPV models for turbofan engine transfer functions (Reberga et al., 2005), and in this paper we use these models to validate our gain-scheduling design procedure.

\section{PROBLEM STATEMENT}

We consider a continuous SISO open-loop plant modeled by a transfer function $G(s, \theta)=B(s, \theta)$ / $A(s, \theta)$ where $A$ and $B$ are polynomials in the Laplace indeterminate $s \in \mathbb{C}$, both parametrized in a vector $\theta \in \mathbb{R}^{p}$, capturing the evolution of the model with respect to its environment. We assume that $\theta$ is a time-varying vector of exogeneous variables that can be measured in real-time. It belongs to a semialgebraic set

$$
\Theta=\left\{\theta \in \mathbb{R}^{q}: g_{i}(\theta) \geq 0, i=1, . . r\right\}
$$

where $g_{i}$ are given multivariate polynomials.

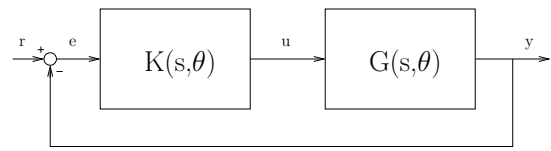

Fig. 1. Negative feedback configuration

Let $K(s, \theta)=Y(s, \theta) / X(s, \theta)$ be a controller depending also on $\theta$. This controller is placed in a negative feedback configuration as shown in

\footnotetext{
1 Snecma is, within the SAFRAN Group, dedicated to design, development and production of engines for civil, military aircraft, launch vehicles and satellites.
}

Figure 1. We outline that neither the order of the controller nor its structure depend on the openloop plant order or structure.

We denote by $M(s, \theta)=N(s, \theta) / D(s, \theta)$ any closed-loop transfer function with this configuration. $M$ can be the closed-loop transfer function $B Y /(A X+B Y)$ between reference signal $r$ and output signal $y$, or the sensitivity transfer function $A X /(A X+B Y)$ between $r$ and error signal $e$. Note that in any case, $M$ can be represented by two polynomials where the numerator and the denominator are both linear in controller polynomials $X$ and $Y$.

With these notations, the problem we are to solve can be expressed as follows.

LPV design problem: Given open-loop plant $G(s, \theta)$, find controller $K(s, \theta)$ such that the closed-loop system $M(s, \theta)$ is stable and its $H_{\infty}$ norm is less than a given bound $\gamma$, for all $\theta$ in $\Theta$.

To address this LPV design problem, we decompose it into three steps:

(1) Sufficient LMI conditions are derived to guarantee stability of a polynomial.

(2) These conditions are extended to robust stability of a polynomial affected by additive unstructured norm-bounded uncertainty.

(3) The conditions are further extended to the case of polynomials with coefficients depending polynomially on $\theta$.

\section{LPV PROBLEM}

In the sequel, we associate to any polynomial $R(s)=\sum_{i=0}^{d} r_{i} s^{i}$ of degree $d$ the vector of its coefficients $R=\left[\begin{array}{llll}r_{0} & r_{1} & \ldots & r_{d}\end{array}\right]$.

\subsection{Stability of a polynomial}

In this paper a stable polynomial is a polynomial with roots in the open left half-plane.

Consider a transfer function $M(s)=N(s) / D(s)$, with no dependence on parameter $\theta$, where $D(s)=\sum_{i=0}^{d} d_{i} s^{i}$ and $N(s)=\sum_{i=0}^{d} n_{i} s^{i}$ are polynomials of degree $d$. We define $y_{M}(t)$ and $u_{M}(t)$ as the output and input signals of this transfer function so that: $Y_{M}(s) / U_{M}(s)=N(s) / D(s)$, where $U_{M}(s)$ and $Y_{M}(s)$ are respectively the Laplace transforms of signals $u_{M}(t)$ and $y_{M}(t)$.

Sufficient conditions for stability of this transfer function are now given, based on Lyapunov's stability theory (de Oliveira and Skelton, 2001). An alternative proof based on polynomials positivity can be found in (Henrion et al., 2003a; Henrion et al., 2003b). 
Define a state-vector $x(t)=\left[y_{M} ; d y_{M} / d t ; \ldots\right.$ $\left.d^{d-1} y_{M} /(d t)^{d-1}\right]$ and $\xi(t)=\left[x ; d^{d} y_{M} /(d t)^{d}\right]$.

Let

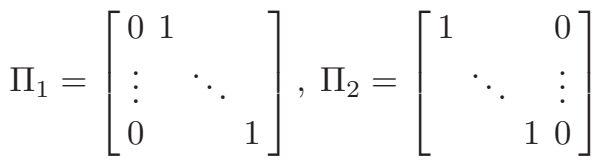

be $d$-by- $(d+1)$ matrices so that: $x(t)=\Pi_{2} \xi(t)$ and $\dot{x}(t)=\Pi_{1} \xi(t)$. Consider a quadratic Lyapunov function $V$ such that : $V(t)=x^{*}(t) P x(t)=$ $\xi^{*}(t) \Pi_{2}^{*} P \Pi_{2} \xi(t)$ and where the star denotes transposition. Finally, define the linear mapping $F(P)=$ $-\Pi_{1}^{*} P \Pi_{2}-\Pi_{2}^{*} P \Pi_{1}$.

Theorem 1. Given a stable polynomial $C(s)$, polynomial $D(s)$ is stable if there exists a symmetric matrix $P$ such that:

$$
C^{*} D+D^{*} C-F(P) \succeq 0
$$

where $\succeq 0$ stands for positive semidefinite, i.e all eigenvalues real nonnegative.

Polynomial $C(s)$ is called the central polynomial. It plays a key role, see section 4.1.

Proof of Theorem 1: It closely follows (de Oliveira and Skelton, 2001). First, for zero initial conditions, recall that asymptotic stability of the dynamical system described in the Laplace domain by the algebraic relation $D(s) Y_{M}(s)=$ $N(s) U_{M}(s)$ depends only on the roots of polynomial $D(s)$, which must belong to the open left half-plane.

Following Lyapunov's second approach, asymptotic stability of this system is also equivalent to the sign conditions $V(t)>0$ and $\dot{V}(t) \leq 0$ along system trajectories, i.e. for all vectors $\xi(t)$ such that $D \xi(t)=0$. By construction of the linear mapping $F$, the time-derivative of the Lyapunov function is given by $\dot{V}(t)=-\xi^{*}(t) F(P) \xi(t)$. Hence the stability conditions can be summarized as:

$$
\begin{array}{ll}
V>0 & \Longleftrightarrow \xi^{*} P \xi>0 \\
\dot{V} \leq 0 & \Longleftrightarrow \xi^{*} F(P) \xi \leq 0
\end{array}
$$

Along system trajectories $\Longleftrightarrow D \xi=0$.

Applying Finsler's Lemma to the last two quadratic inequalities, it follows that there exists a vector $C$ (same dimensions as $D$ ) such that:

$$
\begin{gathered}
C^{*} D+D^{*} C-F(P) \succeq 0 \\
P \succ 0 .
\end{gathered}
$$

The condition $P \succ 0$ can be removed since $C(s)$ is stable, see (Henrion et al., 2003a).

\section{2 $H_{\infty}$ performance}

Suppose now that polynomial $D(s)$ is affected by an additive norm-bounded uncertainty: $D_{\Delta}(s)=$ $D(s)+\Delta N(s),\|\Delta\|_{\infty} \leq \gamma^{-1}$, where $\Delta$ is a matrix of unstructured uncertainty and $\gamma$ is a given positive scalar.

According to the small-gain theorem (Skogestad and Postlethwaite, 1996), robust stability of polynomial $D_{\Delta}(s)$ is equivalent to the $H_{\infty}$ performance constraint:

$$
\left\|N(s) D^{-1}(s)\right\|_{\infty}<\gamma .
$$

Theorem 2. Given a stable polynomial $C(s)$, the transfer function $N(s) / D(s)$ is stable and satisfies the $H_{\infty}$ performance constraint (3) if there exists a symmetric matrix $P$ and a scalar $\lambda$ such that:

$$
\left[\begin{array}{cc}
C^{*} D+D^{*} C-F(P)-\lambda C^{*} C & N^{*} \\
N & \lambda \gamma^{2} I_{n}
\end{array}\right] \succeq 0 .
$$

Proof of Theorem 2: The proof is derived in two steps. Applying first Theorem 1 to uncertain polynomial $D_{\Delta}(s)$ yields the uncertain LMI

$$
C^{*}(D+\Delta N)+\left(D^{*}+N^{*} \Delta^{*}\right) C-F(P) \succeq 0
$$

or equivalently, the quadratic inequality

$\xi^{*}\left(C^{*}(D+\Delta N)+\left(D^{*}+N^{*} \Delta^{*}\right) C-F(P)\right) \xi \geq 0$

that must hold for all vectors $\xi$.

Denoting $z=\Delta^{*} C \xi$, this can be expressed as:

$$
\left[\begin{array}{l}
\xi \\
z
\end{array}\right]^{*}\left[\begin{array}{cc}
C^{*} D+D^{*} C-F(P) & N^{*} \\
N & 0
\end{array}\right]\left[\begin{array}{l}
\xi \\
z
\end{array}\right] \geq 0 .
$$

Moreover, the assumption on the complex uncertainty block $\|\Delta\|_{\infty} \leq \gamma$ can be rewritten as

$$
\gamma^{-2} I-\Delta \Delta^{*} \succeq 0
$$

or equivalently, as the quadratic inequality

$$
\xi^{*} C^{*} C \xi-\gamma^{2} z^{*} z \geq 0 .
$$

Then, applying, the S-procedure (de Oliveira and Skelton, 2001) on quadratic inequalities (5) and (6) yields robust LMI (4). $\square$

\subsection{Extension to LPV systems}

Suppose now that polynomials $N(s)$ and $D(s)$ depend also polynomially on a vector of parameter $\theta$ defined as in Section 2. We denote this dependence as follows:

$D(s, \theta)=\sum_{\alpha \in \mathbb{N}^{q}} D_{\alpha}(s) \theta^{\alpha}, \quad N(s, \theta)=\sum_{\alpha \in \mathbb{N}^{q}} N_{\alpha}(s) \theta^{\alpha}$ 
where $D_{\alpha}(s)$ and $N_{\alpha}(s)$ are polynomials of degree $d$ in $s$.

Recall that polynomials $N(s)$ and $D(s)$ depend linearly on the coefficients of controller polynomials $X(s)$ and $Y(s)$, which are our decision variables. Let us gather these variables into a vector $k$, together with the entries of matrix $P$ and scalar $\lambda$. Inequality (4) becomes an LMI in $k$ polynomially parametrized in $\theta$ :

$$
\begin{aligned}
& L(k, \theta)=\sum_{\alpha} L_{\alpha}(k) \theta^{\alpha}= \\
& {\left[\begin{array}{cc}
C^{*} D(\theta)+D(\theta)^{*} C-F(P)-\lambda C^{*} C & N(\theta)^{*} \\
N(\theta) & \lambda \gamma^{2} I_{n}
\end{array}\right] \succeq 0 .}
\end{aligned}
$$

The problem here is to find parameter $k$ ensuring positive semidefiniteness of this matrix inequality for all values of parameter $\theta$ in $\Theta$. This is a parametrized LMI problem, a semi-infinite convex optimization problem which is difficult to solve in general. Several numerical methods have been proposed recently, see for instance the survey (Scherer, 2006). The technique presented here is based on a matrix extension of a representation result proposed by Putinar for polynomial positive on semialgebraic sets.

We make the non-restrictive assumption that the semialgebraic set $\Theta$ is compact and that it is included in a ball of radius $\rho$ centered around the origin, so that in the description (1) we can add the redundant constraint $g_{r+1}(\theta)=\rho^{2}-\|\theta\|_{2}^{2} \geq 0$.

Lemma 1. There exists $k$ such that $L(k, \theta) \succ 0$ for all $\theta \in \Theta$ if and only if there exist sumof-squares matrix polynomials $M_{i}(\theta)$ such that $L(k, \theta)=M_{0}(\theta)+\sum_{i=1}^{r+1} g_{i}(\theta) M_{i}(\theta)$.

Proof of Lemma 1: See Theorem 24 in (Scherer, 2006), an extension to the matrix case of a scalar result by Putinar used for polynomial optimization in (Lasserre, 2001).

The discrepancy between the strict inequality in Lemma 1 and the non-strict inequality (7) is not relevant numerically speaking since $L(k, \theta) \succ 0$ can be written as $L(k, \theta) \succeq \epsilon I$ for an arbitrarily small positive scalar $\epsilon$.

Checking if a multivariate matrix polynomial of given degree is a sum-of-squares amounts to solving an LMI problem (Scherer, 2006), but we do not know in advance the degree of the polynomials $M_{i}(\theta)$. So in practice solving parametrized LMI (7) amounts to solving a hierarchy of LMI of increasing size, in the same spirit as in (Lasserre, 2001).

\subsection{Remarks}

Discrete-time systems could also be considered, via an appropriate change of the linear mapping $F(P)$. See (Henrion et al., 2003a; Henrion et al., 2003b) for instance.

Since the Lyapunov matrix $P$ is chosen to be constant, LMIs (7) ensure quadratic stability (Scherer and Weiland, 2000). It could also depend on $\theta$. In that case, the polynomial matrix $L(k, \theta)$ in $(7)$ is slightly modified but the rest of the approach is valid.

Other representations for matrix polynomials positive semidefinite on semialgebraic sets can be used in Lemma 1, see (Scherer, 2006) for a survey. These representations provide sufficient finitedimensional LMI conditions for solving polynomially parametrized LMI on compact sets, and necessity is recovered only asymptotically, i.e. for multipliers of arbritrary large degree.

Although the approach is presented for SISO transfer functions, it is worth mentioning that it is still relevant for MIMO (Multiple Input Multiple Output) systems. The only additional assumption is to consider MIMO transfer functions with scalar denominators. Indeed, in the general MIMO case, as the product of matrices is not commutative, in a closed-loop transfer $M(s, \theta)$ the polynomial matrices $X(s)$ and $Y(s)$ of the controller do not typically enter affinely the numerator and the denominator. Choosing scalar denominators is nonrestrictive: one can always take the least common multiple of denominators of the scalar transfer functions.

\section{APPLICATION TO A TURBOFAN ENGINE}

We now apply this technique to the control of a turbofan engine. In (Henrion et al., 2004; Reberga et al., 2005) we obtained LPV models of different loops of a turbofan. The models depend on the scalar variable $\theta=\mathrm{P} S 32$, representing the pressure in the combustion chamber. In this paper we use two of these models to study two particular transfers. The first one involves the fuel flow input WF32 and the compressor speed XN25. The second is between WF32 and the low pressure fan speed XN2.

The LMI problems were parsed by the YALMIP interface 3.0 (Löfberg, 2001) which handles polynomial matrix sum-of-squares problems. Resulting semidefinite programs were solved by SeDuMi 1.1 (Sturm, 1999) on Matlab 6.5. LPV controllers are validated on a nonlinear Simulink turbofan engine model provided by Snecma. Different points of the flight envelope (altitude and Mach number 
combinations) can be chosen to check robustness of the system. Due to saturation limits for WF32 a basic anti-windup scheme (Gomes da Silva Jr. and Tarbouriech, 2005) is added for the validations. For confidentiality reasons, the units and scales do not appear on the figures.

Before presenting these applications, we focus on the tuning of the central polynomial which is the key ingredient of the approach.

\subsection{Central polynomial tuning}

As mentioned in (Henrion et al., 2003b), it is hard to give a general formula for the choice of the central polynomial. Somehow, it can be compared to the choice of the weighting filters of the mixed sensitivity problem, see (Skogestad and Postlethwaite, 1996) for example. The only necessary condition is to choose a stable polynomial.

In this paper, we try to show the impact of this parameter on the closed-loop system behaviour. Although it is difficult to prove theoretically that $C(s)$ can be considered as a possible pole placement, Figure 2 shows that there is a genuine link between the roots of the central polynomial and the poles of the closed-loop system: the poles of the LPV closed-loop system tend to be close to the roots chosen for $C(s)$. On each of the root locii we represent the migration of 4 closed-loop poles for various operating points, that is, various values of the scheduling parameter $\theta$.
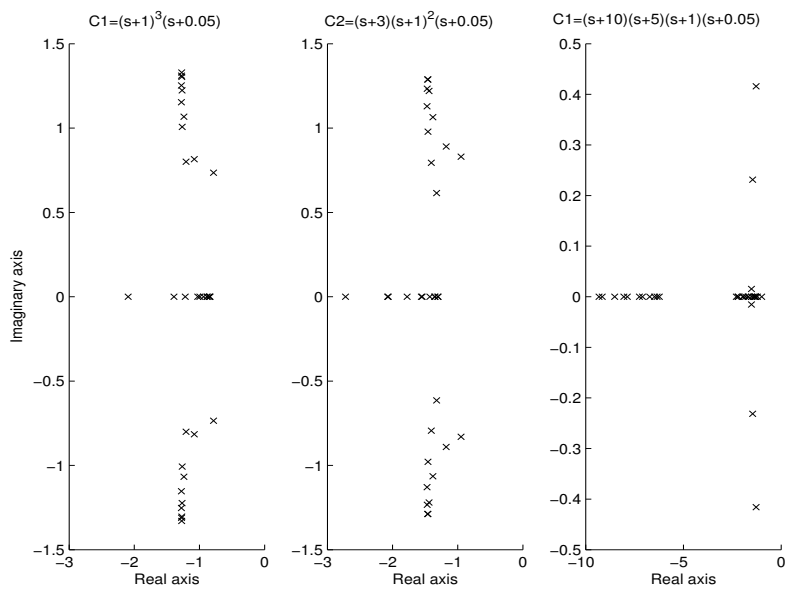

Fig. 2. Compared closed-loop system poles for various operating points and three different central polynomials.

In the case of an application, a good knowledge of the process is also useful. Keeping in mind the natural good dynamics of the model gives indications on the desired closed-loop system and so on the roots of the central polynomial.

Recall that the degree of $C(s)$ is equal to the degree of the denominator $D(s, \theta)$ of the transfer function. When the degree of the denominator increases, so is the number of roots of $C(s)$ to be chosen. This gives more degrees of freedom, but the tuning is necessarily more involved. Also the LMIs are larger, hence potentially more difficult to solve.

Undoubtedly, these indications remain vague. As explained before, it is the designer's task to choose the adequate central polynomial. Since there is no criterion over $C(s)$ to optimize in the design LMI, several iterations are necessary to find out a consistent central polynomial. This is the key to a successful synthesis.

\subsection{WF32-XN25 loop}

The polynomial approach is applied to control a second order model with an affine dependence on $\theta$. We seek an LPV PI controller, whose transfer function can be written:

$$
K(s, \theta)=\left(k_{i 1}+k_{i 2} \theta\right) \frac{1}{s}+\left(k_{p 1}+k_{p 2} \theta\right) .
$$

Closed-loop simulations of the LPV system are shown on Figure 3. The corresponding variations of $\theta$ are presented on Figure 4.

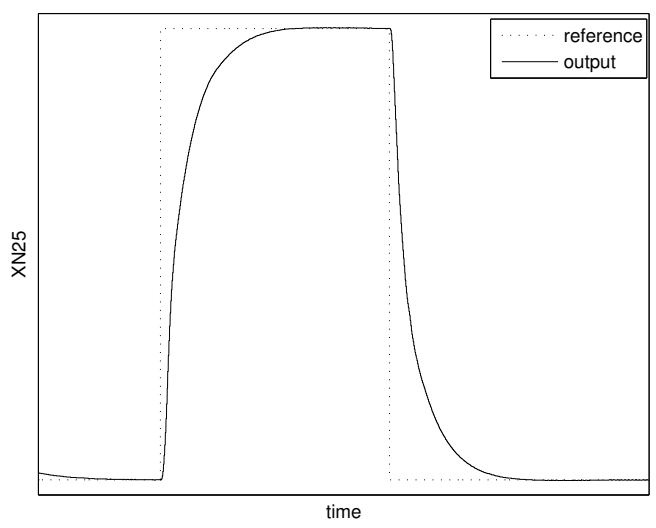

Fig. 3. Control of the WF32-XN25 loop. Step response. Altitude: 10000ft, Mach: 1.3.

Tracking is ensured by the integrator in the controller, and the time-response behavior is monitored by a suitable choice of $C(s)$.

\subsection{WF32-XN2 loop}

We consider a first order open-loop LPV model of this loop with a second order dependence on $\theta$. We control it with a second order LPV controller.

Figure 5 represents the closed-loop system for a variation of parameter $\theta$ given by Figure 6 . 


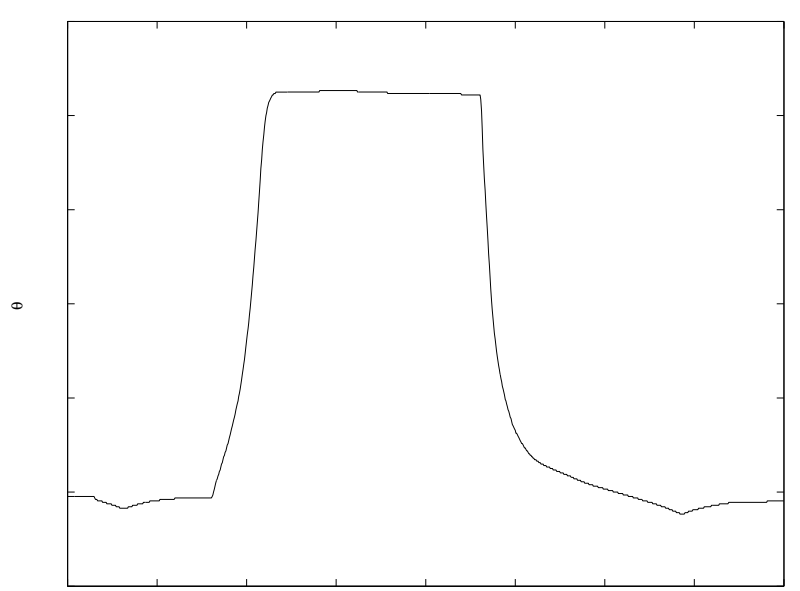

time

Fig. 4. Control of the WF32-XN25 loop. Evolution of the scheduling parameter $\theta$.

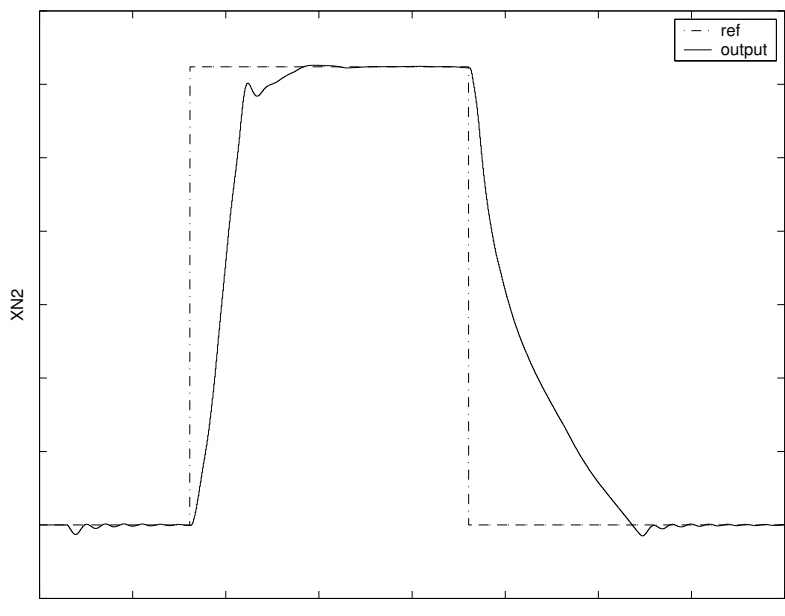

Fig. 5. Control of the WF32-XN2 loop. Step response. Altitude: 0ft, Mach: 0.

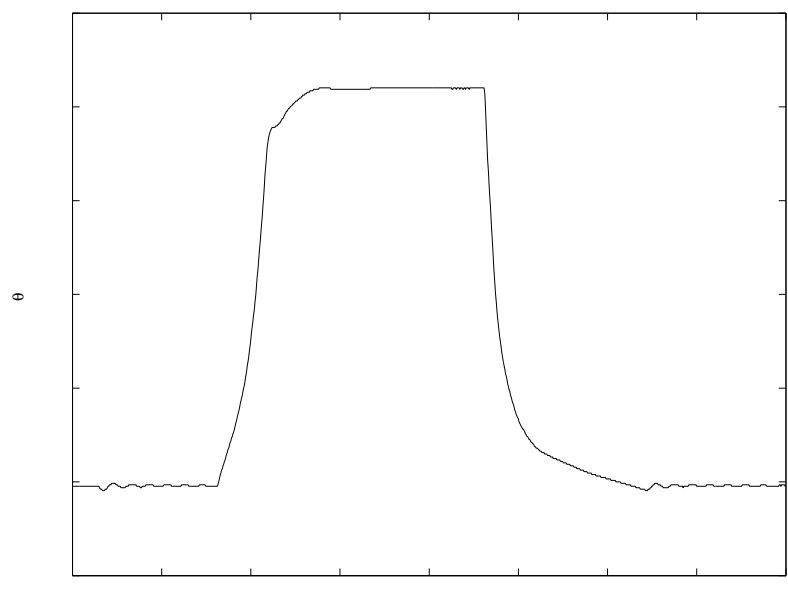

time

Fig. 6. Control of the WF32-XN2 loop. Evolution of the scheduling parameter $\theta$.

\section{CONCLUSION}

We have described an extension of the fixedorder controller design procedure of (Henrion et $a l ., 2003 b)$ to SISO gain-scheduling design with guaranteed $H_{\infty}$ performance over the whole parameter range. We used the idea of central polynomial developed in (Henrion et al., 2003b) in order to derive an LMI formulation with the following characteristics:

- the controller variables appear explicitly as decision variables in the design LMI, thus avoiding the use of tedious linear algebra to reconstruct controller parameters;

- the order and the structure of the controller can be fixed from the outset, independently of the open-loop system order and weighting functions;

- the design conditions are directly formulated in the polynomial setting, using polynomial matrix fraction descriptions;

- we allow polynomial dependence of the openloop system and controller transfer functions in the scheduling parameters;

- a decoupling between the Lyapunov variables and the controller variables allows the use of parameter-dependent Lyapunov functions.

The gain-scheduling design procedure was validated in the scope of an on-going industrial research project on aircraft turbofan engine control. It is integrated within the ATOL software framework developed by Snecma (Vary and Reberga, 2005).

The main difficulty of the approach is of course the appropriate choice of the central polynomial. Roughly speaking, as explained in (Henrion et $a l ., 2003 b)$, the central polynomial enforces the pole dynamics desired in closed-loop. For a given choice of a central polynomial, if the design LMI are infeasible, then it may mean that the desired closed-loop dynamics are not achievable. Unfortunately, it may also happen that the desired dynamics are achievable, but that the design LMI are too conservative to retrieve the appropriate controller. This is the main limitation of the whole approach.

We do not expect difficulties when extending the approach to MIMO systems, as soon as we preserve linearity of the numerator and denominator polynomials in the controller coefficients.

Open issues and future research directions related with this work can be sketched as follows:

- better characterize the impact of the choice of the central polynomial on the achievable closed-loop performance;

- integrate time-domain constraints (overshoot, saturation) and/or combine with anti-windup design in the scope of aircraft turbofan engine control; 
- study numerical aspects related with conditioning of the design LMI, study alternative choices of polynomial bases.

\section{ACKNOWLEDGMENTS}

This work on LPV design of turbofan fan engines was initiated during Luc Reberga's PhD thesis at LAAS-CNRS (2002-2005), in collaboration with Florian Vary at Snecma. It benefited significantly from technical input by Luc and Florian, whose contribution is gratefully acknowledged. Didier Henrion also acknowledges lively and constructive discussions on LMIs, positive polynomials and LPV systems with Mauricio de Oliveira, Pedro L. D. Peres and Carsten Scherer. The research of Didier Henrion was funded in part by projects No. 102/05/0011 and No. 102/06/0652 of the Grant Agency of the Czech Republic and project No. ME 698/2003 of the Ministry of Education of the Czech Republic.

\section{REFERENCES}

Balas, G. J. (2002). Linear parameter-varying control and its application to a turbofan engine. Int. J. of Robust and Nonlinear Control 12(9), 763-796.

Bruzelius, F. (2004). Linear parameter-varying systems - An approach to gain scheduling. $\mathrm{PhD}$ thesis. Chalmers University. Göteborg, Sweden.

de Oliveira, M. C. and R. E. Skelton (2001). On stability tests for constrained linear systems. pp. 241-257. Lecture Notes in Control and Information Sciences. Springer Verlag. Edited by S. O. Reza Moheimani.

Gomes da Silva Jr., J. M. and S. Tarbouriech (2005). Anti-windup design with guaranted regions of stability: an LMI-based approach. IEEE Trans. on Automatic Control 50(1), 106-111.

Henrion, D., D. Arzelier and D. Peaucelle (2003a). Positive polynomial matrices and improved LMI robustness conditions. Automatica 39(8), 1479-1485.

Henrion, D., L. Reberga, J. Bernussou and F. Vary (2004). Linearization and identification of aircraft turbofan engine models. In: IFAC symposium on Automatic Control in Aerospace. St Petersburg, Russia.

Henrion, D., M. Šebek and V. Kučera (2003b). Positive polynomials and robust stabilization with fixed-order controllers. IEEE Trans. on Automatic Control 48(7), 1178-1186.

Lasserre, J.-B. (2001). Global optimization with polynomials and the problem of moments. SIAM J. on Optimization 11(3), 796-817.
Leith, D.J. and W.E. Leithead (2000). Survey of gain-scheduling analysis and design. Int. J. Control 73(11), 1001-1025.

Löfberg, J. (2001). YALMIP: Yet Another LMI Parser. ETH Zurich, Switzerland.

Reberga, L., D. Henrion, Bernussou J. and F. Vary (2005). LPV modeling of a turbofan engine. In: IFAC World Congress on Automatic Control. Prague, Czech Republic.

Rugh, W.J. and J.S. Shamma (2000). Research on gain scheduling. Automatica 36, 1401-1425.

Scherer, C. W. (2006). LMI relaxations in robust control. European J. of Control 12(1), 3-29.

Scherer, C. W. and S. Weiland (2000). Linear Matrix Inequalities in Control. DISC Course Lecture Notes. The Netherlands.

Skogestad, S. and I. Postlethwaite (1996). Multivariable feedback control. Wiley, Londres.

Sturm, J.F. (1999). Using SeDuMi 1.02, a Matlab toolbox for optimization over symmetric cones. Optimization Methods and Softwares 11(12), 625-653.

Vary, F. and L. Reberga (2005). Programming and computing tools for jet engine control design. In: IFAC World Congress on Automatic Control. Prague, Czech Republic.

Wassink, M. G., M. van de Wal, C. W. Scherer and O. Bosgra (2005). LPV control for a wafer stage: beyond the theoretical solution. Control Engineering Practice 13, 231-245. 